\title{
The use of bone marrow derived mesenchymal stem cell for cornea regeneration in rabbit model
}

\author{
Kong Yong Then ${ }^{1,2}$, M. Azlina ${ }^{3}$, A.R. Ropilah², B.H.I. Ruszymah ${ }^{4,5}$, \\ C.M. Rohaina ${ }^{4}$, M.H. Ng$^{4}$ \\ 'International Specialist Eye Centre Sdn. Bhd, The Boulevard, Mid Valley City, \\ Kuala Lumpur, Malaysia; ${ }^{2}$ Department of Ophthalmology, Universiti Kebangsaan \\ Malaysia Medical Centre, Kuala Lumpur, Malaysia; ${ }^{3}$ Faculty of Medicine and \\ Health Science, Universiti Sains Islam ,Kuala Lumpur, Malaysia; ${ }^{4}$ Tissue Engineering \\ Centre, Universiti Kebangsaan Malaysia Medical Centre, Kuala Lumpur,Malaysia; \\ ${ }^{5}$ Department of Physiology, Faculty of Medicine, Universiti Kebangsaan Malaysia, \\ Kuala Lumpur, Malaysia
}

\begin{abstract}
Aim or purpose: To evaluate the use of autologous bone marrow derived mesenchymal stem cells (BM-MSCs) to treat cornea stromal defect in a rabbit model.

Methods: A non-randomized interventional controlled animal study involving twenty-one adult New Zealand white rabbits. Corneal deep lamellar dissections were created in three groups of rabbits and treated accordingly. Group I: Autologous bone marrow derived MSCS with autologous fibrin and human amniotic membrane. Group II: Autologous fibrin with human amniotic membrane without MSCs. Group III: No treatment. Clinical outcome was evaluated by corneal re-epithelization, corneal opacity, corneal thickness and histology. Results: BM-MSCs were successfully isolated from bone marrow of seven rabbits based on the adherence property of the cells to the plastic of the cell culture plate. At day 60 corneal thickness was significantly thicker in Group I. The localization of PKH26-labeled $B M-M S C s$ showed an increase in cell density at the transplanted site, proving its role in cornea stromal regeneration. Although the cornea clarity was not achieved in this study, we believe that cornea stromal remodeling requires many months to years to regain its original optical quality.

Conclusion: Locally transplanted BM-MSCs may be a useful source for cornea stromal regeneration. The use of autologous BM-MSCs offers a promising option for treating corneal disorder without the risk of immune-rejection and calcification.
\end{abstract}

Keywords: BMSCs, corneal stroma, rabbit, tissue engineering, transplantation

\section{Introduction}

The cornea is damaged in various diseases, such as trauma and injuries. Corneal melt is a debilitating disease resulting in severe loss of vision. Such cases usually result in scarred and vascularized corneas or evisceration. At present, the only treatments available are inorganic tissue glue or corneal transplantation. However, the main risk of corneal transplantation in an inflamed eye is tissue rejection. Furthermore, in many countries access to corneal tissue is severely hampered by the lack of organ donors. The use of inorganic tissue glue is also hampered by the fact that

Correspondence: Kong Yong Then, Universiti Kebangsaan Malaysia Medical Centre, Jalan Yaacob Latif, Bandar Tun Razak, 56000, Cheras, Kuala Lumpur, Malaysia.

E-mail: kythen@hotmail.com 
they are very toxic to the underlying cell. This may sometimes result in a larger corneal hole than before. Using a person's own cells in corneal regeneration may address such problems.

Autologous corneal tissue engineering is a promising therapeutic approach to overcome the problems of primary immune rejection and the limitation of corneal donors. Norzana et al. ${ }^{9}$ reported that corneal epithelium can be serially expanded in serum-free and feeder layer-free culture system, and be used for corneal tissue engineering. In clinical cases, the epithelial cells can be obtained from donors or from the healthy eye of the same patient. However, the former may cause tissue rejection, whilst the latter may not be suitable in cases where both eyes are damaged.

A recent report suggested that transplantation of human mesenchymal stem cells could reconstruct the damaged cornea. ${ }^{8,12,14}$ Ye et al. ${ }^{14}$ showed that systemically transplanted MSCs can engraft to an injured cornea and promote wound healing by differentiation, proliferation and synergizing with hematopoietic stem cells. The therapeutic effect of the transplantation may be associated with the inhibition of inflammation and angiogenesis. However, these studies are mainly looking at cornea epithelial regeneration as opposed to stromal regeneration. The latest study by Gu et al. ${ }^{5}$ showed MSCs could differentiate into corneal epithelial like cells in vivo and ex vivo. However, most corneal diseases involve the corneal stroma, which accounts for $90 \%$ of corneal thickness. Diseases that can cause cornea melt include autoimmune keratitis, infection and ectatic diseases. Arnalich-Montiel et al. ${ }^{1}$ did a study to look at stroma regeneration. Their results showed that adiposederived stem cells could be a source for cellular therapy for the corneal stroma.

Barbosa and co-workers ${ }^{2}$ suggested that corneal myofibroblasts, which make up the corneal stromal layers, can be derived from MSCs in a chimeric mice study. MSCs also have the capacity to differentiate to corneal stromal cells when the cells were intravenously introduced, as reported by Harada. ${ }^{6}$ A recent study by Park et al. ${ }^{11}$ reported that MSCs are able to be differentiated to keratocyte-like cells in vitro by using keratocyte conditioned-medium (KCM).The MSCs differentiated keratocytelike cells expressed both keratocan and ALDH1A1 and consistently upregulated lumican. This study showed that conditioned medium promotes differentiation of human MSCs to corneal keratocyte-like cells in vitro.

The ability to bioengineer a person's own cornea using autologous MSCs in improving visual outcome will represent a significant improvement in corneal tissue engineering. This may delay or reduce the need for corneal transplantation and improves the patient's quality of life. It may also prevent the risk of corneal rejection, which is the main cause of graft failure. It also avoids the controversy surrounding the use of embryonic stem cell. The primary aim of a bioengineered cornea is to overcome the undesirable outcome of corneal melt following insult and the secondary aim is to restore the corneal thickness and clarity.

The objective of this study was to evaluate the use of autologous bone marrow derived mesenchymal stem cells (BM-MSCs) to treat cornea stromal defect in a rabbit model. 


\section{Materials and methods}

\section{Animals experiment approval}

This study involved 21 adult male New Zealand white rabbits weighing $1.8-2.3 \mathrm{~kg}$, obtained from Medical Faculty UKM Animal Unit after the approval of Universiti Kebangsaan Malaysia Animal Ethics Committee. They were treated according to the Guide for The Care and Use of Laboratory Animals (National Academy of Science 1996). The animals were randomly divided into three groups of seven rabbits each. Group I: Autologous BM-MSCs with autologous fibrin glue and human amniotic membrane (HAM); Group Il: Autologous fibrin glue with human amniotic membrane without BM-MSCs; Group III: No treatment. Bone marrow was harvested from the left iliac crest bone in all the rabbits in group I. Four $\mathrm{ml}$ of blood were collected to produce autologous fibrin from all the rabbits in group I and II.

\section{Isolation, expansion and labeling of BM-MSCS}

Autologous BM-MSCs aspiration from the Group I was harvested and expanded in the laboratory using widely accepted protocol and commercially available tissue culture media. ${ }^{13}$ The animals were being anaesthetized by intramuscular injection of a mixture of zoletil $50 \mathrm{mg} / \mathrm{kg}$ (Virbac), ketamine hydrochloride $50 \mathrm{mg} / \mathrm{kg}$ (Bioketan) and $10 \mathrm{mg} / \mathrm{kg}$ of xylazine (Troy Lab) $(0.2 \mathrm{ml} / \mathrm{kg}$ body weight). Once they were sedated, the animals were then placed in a wooden box restrainer. Intravenous animal anesthetic drugs regime $(0.1 \mathrm{ml} / \mathrm{kg}$ body weight of the mixture) was given through the lateral marginal ear vein to ensure the animals were deeply sedated. Left iliac crest region of the rabbit was shaved, painted and draped. One centimeter skin incision was made over the iliac crest. Four $\mathrm{ml}$ of rabbit bone marrow was harvested using a percutaneous $18-\mathrm{G}$ needle aspiration from the iliac crest. The bone marrow was kept in vacutainers containing $0.1 \mathrm{ml}$ of heparin $(3000 \mathrm{U} / \mathrm{ml}-\mathrm{B}$ / Braun) at room temperature and transferred to the cell culture facility.

Isolation and expansion of BM-MSCs were performed as previously described. ${ }^{13}$ They were isolated solely based on their plastic adherent property. All cultures were incubated at $37^{\circ} \mathrm{C}$ in a humidified atmosphere of $5 \% \mathrm{CO}_{2}$. Fresh medium was added on the third day. Medium was changed upon substantial cell attachment and later, twice a week in a $75 \mathrm{~cm}^{2}$ flask for the second passage and in a $175 \mathrm{~cm}^{2}$ flask for the third passage. Haematopoietic cells and non-adherent cells were removed along with the media. Upon $80 \%-90 \%$ cell confluency, cells were detached by the addition of $0.05 \%$ trypsin-EDTA (Gibco USA) and viable cells counted using trypan blue dye-exclusion-method. Cells were subsequently sub-cultured at a standard density of 10,000 cells $/ \mathrm{cm}^{2}$. Adherent cells after the third passage referred to as third-generation MSCs, were used for this experiment.

\section{Preparation of autologous fibrin}

An amount of four $\mathrm{ml}$ of fresh rabbit blood was collected through the lateral marginal artery from the rabbit's ear. Blood was collected in sodium citrate tubes followed by rapid inversion of the tube to deter blood from clotting and kept at room temperature. It was then centrifuged at $3000 \mathrm{rpm}$ for five minutes. The plasma 
layer (top yellowish layer) was gently transferred to a new tube with a pipette without disturbing the bottom layer (red layer). This process was repeated three times to ensure complete removal of residual cellular components. The plasma was sterile-filtered using a $0.2 \mu$ syringe filter (Sartorius, USA) to remove the cell debris that may cause spontaneous clotting of the plasma. Plasma was stored at $-20{ }^{\circ} \mathrm{C}$ until the day of transplantation.

\section{Animal model}

The rabbits were anesthetized and the quality of ocular surface was evaluated by the degree of smoothness using slit lamp microscopy (SL-DC 1, Topcon, Japan) with a Topcon anterior segment camera. The cornea thickness was measured by anterior segment optical coherence tomography (OCT-Visante Carl Zeiss). The left eye was cleaned and draped and was kept open by a speculum. Left cornea defect was created by deep lamellar dissection (Fig. 1A-F). A five-mm trephine was used to standardize the size of the defect. A guarded diamond knife was calibrated to a desired corneal thickness. An incision of $80 \%$ to $90 \%$ thickness of the rabbit's cornea was created. The cornea was then dissected using Melles' corneal lamellar dissector and the edge was cut by corneal scissors. The thickness of lamellar dissection was then evaluated by anterior segment OCT and slit lamp to assure the desired corneal thickness was removed.

Fig. 1.
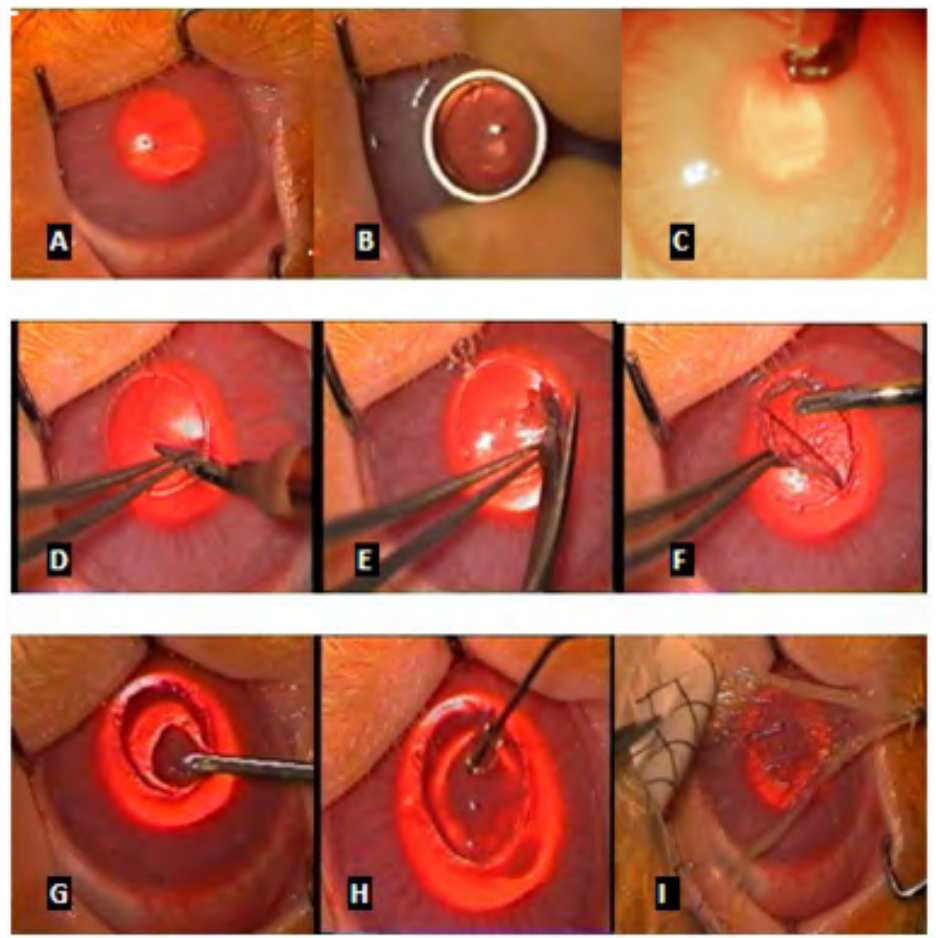


\section{In-vivo transplantation}

Regarding the rabbits in Group I, BM-MSCs were transplanted with an autologous fibrin. On the day of transplantation, monolayer cells were trypsinized and centrifuged at $3000 \mathrm{rpm}$ for five minutes to obtain a cell pellet of approximately ten million cells. Autologous fibrin derived from plasma was used as BM-MSC cell carrier. Stored plasma was thawed on the day of surgery. Calcium chloride ( 0.5 $\mathrm{mmol} / \mathrm{l}$ ) was added to one $\mathrm{ml}$ of plasma to initiate the polymerization process. Cell fibrin mixtures were then filled into the cornea defect. A fresh frozen human amniotic membrane (HAM) was sutured over the site using 10/0 nylon sutures to be held in place for seven days (Fig. 1G-I).

In Group II, corneal defects were treated with the autologous fibrin without cells. As in group I, the treated eyes were covered with HAM. The HAM was removed on day seven post transplantation. Group III consisted of cornea defects without treatment and were left to heal on their own. All rabbits were returned to their cages after the procedure and were allowed to move freely. Topical dexamethasone $0.1 \%$ (Alcon ${ }^{\circ}$ ) and moxifloxacin $0.5 \%$ (Alcon ${ }^{\circ}$ ) were used four times daily for two weeks. Only dexamethasone was continued twice daily for another month to all corneas.

\section{Follow-up and clinical evaluation}

Each eye of the cornea defect underwent slit lamp examination, fluorescein staining and viewing under blue filter, and anterior segment OCT at postoperative day $0,7,14,30$, and 60 . The corneal epithelial integrity was quantified by the ratio of the epithelial defect area to the total cornea using simple Imaging Measurement Software (Topcon, US). The corneal opacity was assessed using published corneal opacity grading technique by visualizing the ocular tissue beneath the cornea. ${ }^{4}$ The corneal clarity was graded as: grade 0 , totally clear with no opacity seen by any method of slit lamp microscopic examination; grade 1, haze of minimal density seen with difficulty with direct and diffuse illumination; grade 2 , mild haze easily visible with direct focal slit illumination; grade 3, moderately dense opacity that partially obscured the iris details; grade 4 , severely dense opacity that completely obscured the details of intraocular structures. Corneal thickness was measured with anterior segment OCT at preoperatively and post operatively day 30 and 60 . Six readings were taken of the center corneal thickness and mean thickness was used for the data.

\section{Statistical analysis}

Epithelial defect size and cornea thickness were compared with repeated measure ANOVA. To compare statistical association of cornea opacity among group, we used the Fisher exact test.

\section{Histology examination and localization of MSC cells in the cornea}

For localization of the BM-MSC cell experiment, one rabbit in group I was euthanized after two weeks and one after four weeks and the remaining were euthanized 60 days after cell transplantation. Several sections of each cornea were stained with hematoxylin and eosin and von Kossa staining for light microscopy examination 
(Olympus BX51; Olympus Co. Ltd., Tokyo, Japan). DAPI stain was used to label all cells nuclei in the corneas in the biosafety experiment. Localization of PKH26 labeled BM-MSC cells using Red Fluorescent Cell Linker Kit (Sigma-Aldrich, Inc. Saint Louis, Missouri, USA) was achieved using confocal microscope (LSM 510 Meta, Zeiss). This technique was performed according to the manufacturer's instruction. Cell tracking was observed at week 2 and 4 , following transplantation.

\section{Result}

The treatment groups were successfully performed, i.e., Group 1: autologous BM-MSCs with autologous fibrin glue and human amniotic membrane (HAM); Group Il: autologous fibrin glue with human amniotic membrane without BM-MSCs; Group III: no treatment. The mean area of baseline epithelial defect ranged from $19.04 \mathrm{~mm}^{2}$ to $19.51 \mathrm{~mm}^{2}$. The statistical analysis revealed there was no significant difference in baseline epithelial defect area among the three groups. This was not surprising as all the defects are created in a well-defined area using a trephine (Fig. 2). All the rabbits showed significant improvement in corneal re-epithelization by day 60 among their group.

Fig. 2.

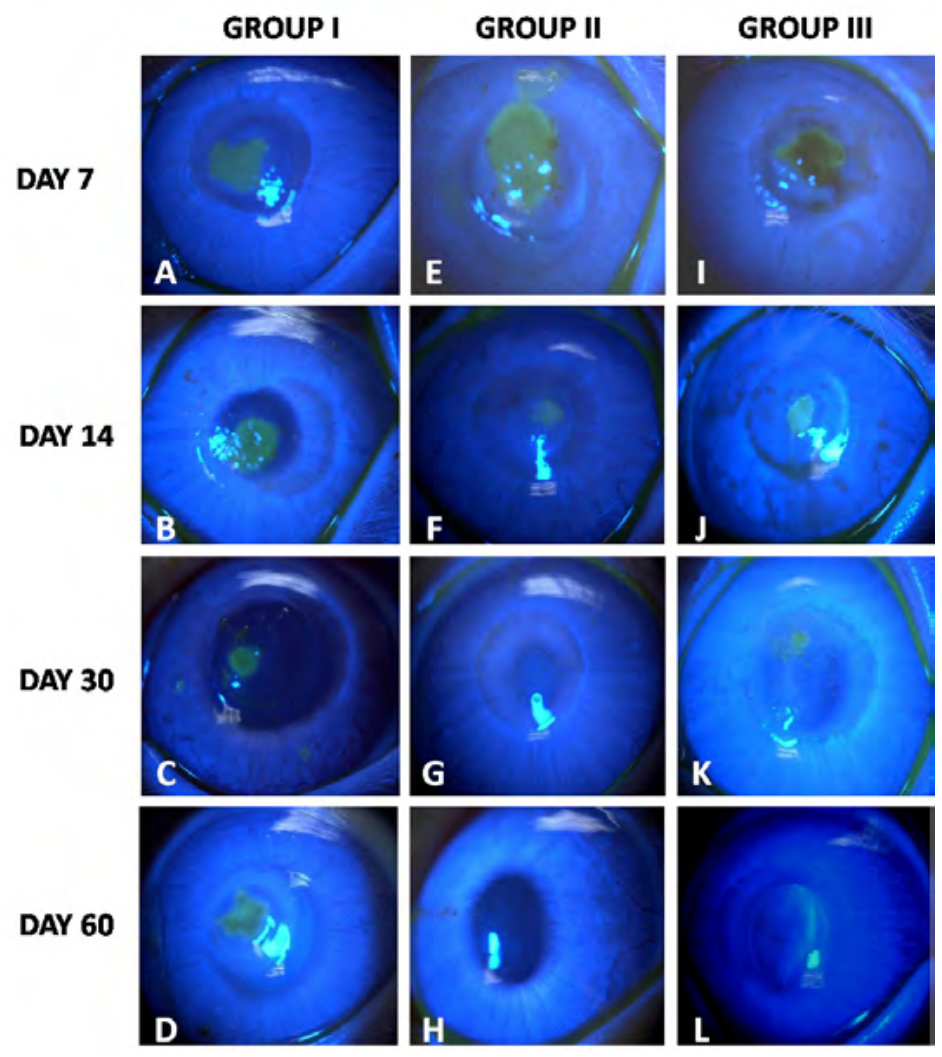


However, overall cornea re-epithelization in the three groups did not show any statistical significance (Table 1). The results showed that BM-MSCs transplantation did not improve the rate of epithelial healing.

Corneal opacity was observed in all three groups during follow-up. Fig. 3 shows a photography of one of the rabbits in each group on day 14, day 30 and day 60 of the follow-up. In group III, the corneas were much clearer and only one rabbit developed moderately dense opacity at day 30 . In addition, the opacities observed in group I tended to be denser at the edge of the dissected cornea, forming a ring appearance. However, at day 30 the corneal opacity in the three groups did not show any statistical significance (Table 2 ).

Fig. 3.

GROUP I
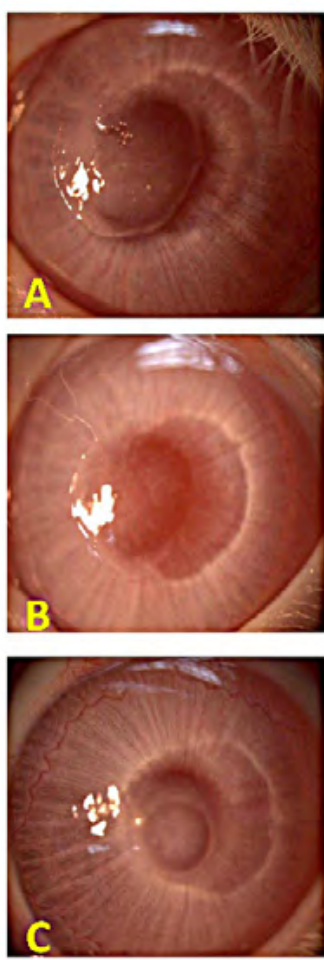

GROUP II
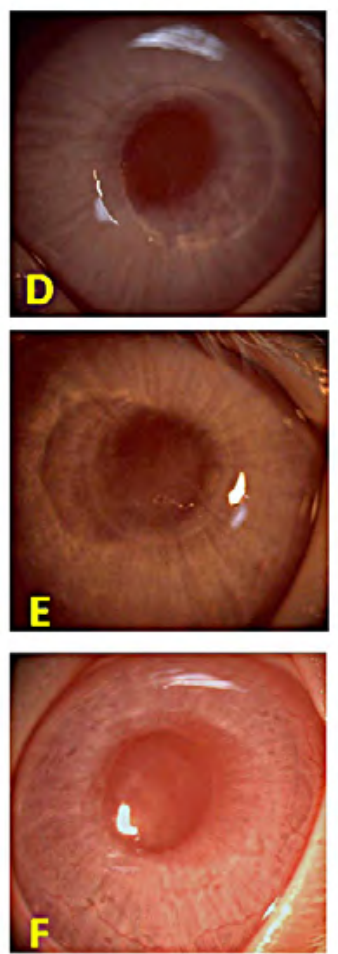

GROUP III
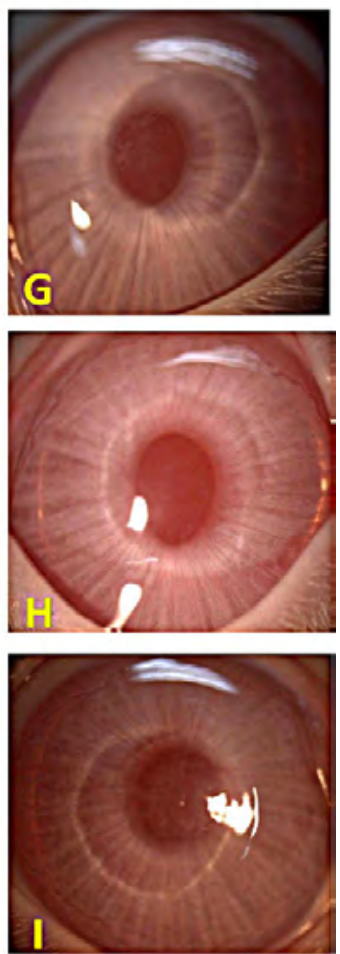

The normal corneal thickness in all rabbits ranges from $405 \mu \mathrm{m}$ to $342 \mu \mathrm{m}$ with mean thickness of $369 \mu \mathrm{m}$. The mean corneal thickness preoperatively in group I: $351 \mu \mathrm{m}$; group II: $372 \mu \mathrm{m}$ and group III: $374 \mu \mathrm{m}$. We removed almost $80 \%$ of corneal thickness during deep lamellar dissection. There appeared to be a significant increase in the corneal thickness in all three groups at day 30 and day 60. At day 60 , the final corneal thickness was also significantly higher in group I $(258.40 \mu \mathrm{m})$ 
compared to group II $(215.33 \mu \mathrm{m})$ and III $(225.60 \mu \mathrm{m})$ (Table 3). With the advantage of high resolution anterior segment $\mathrm{OCT}$, we managed to clearly visualize the depth of the corneal defect and the transplanted BM-MSCs covered with HAM (Fig. 4).

Fig. 4.
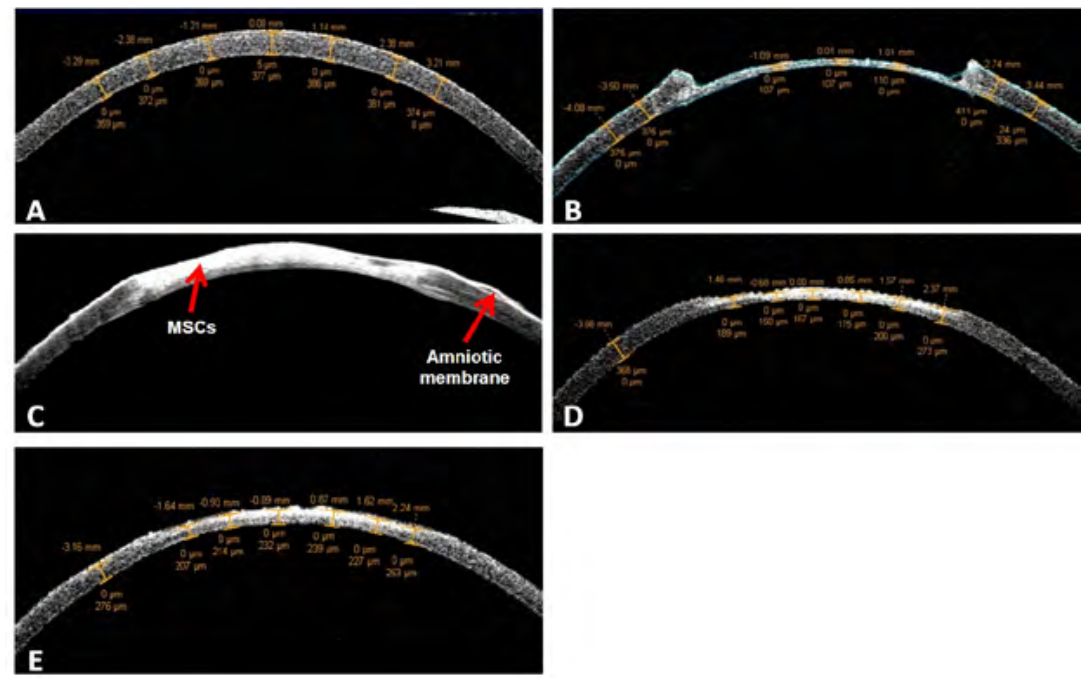

At day 30, a confocal microscope examination of the transplanted cornea which received a PKH26 labelled MSCs showed localization of PKH26 MSCs at the area of defect and edge of the wound (Fig. 5A). Histology staining with Hematoxylin and Eosin in all three groups (Fig. $5 \mathrm{E}-\mathrm{G}$ ) showed that the rabbit's epithelium had completely covered the damage cornea surface with variable epithelium layer. The epithelium in the peripheral corneal areas was normal in appearance with a total thickness of five to seven layers. It was noted that there was an increase in epithelial thickness in group II and III. Stromal regeneration was observed in the group I at the edge of the wound with increase in cellular density compared to group II and III which was lesser toward the centre.

Fig. 5.

\section{Discussion}

Our study was based on clinical observation as an objective measurement. The size of epithelial defect, corneal opacity and corneal thickness were used as parameters for stroma regeneration. In our study, corneal neovascularization was not observed in all three groups. It seemed that neither the MSCs nor fibrin scaffold are capable of angiogenesis.

Epithelial healing rates were the same in all three groups. It seemed that transplantation of BM-MSCs or fibrin alone did not affect the healing rate on epithelium. 
This result differs from previous study in other groups. ${ }^{10,14} \mathrm{~A}$ study by Ma et al. ${ }^{8}$ also showed significant improvement in re-epithelization in MSCs-treated group. The re-epithelization was quantified by less than one fourth of cornea fluorescein staining. It differs from our study method in that we use autologous fibrin as a scaffold and then mixed with autologous MSCs pellets before transplantation. Their method uses amniotic membrane as a cultivating sheet for the MSCs before being transplanted. This may be due to the preservation of stromal layer in their cornea rabbit model whereas in our study, we removed almost $80 \%$ of the stroma layer. We postulated that cellular migration in our study might be inhibited by the corneal construct. Instead of enabling the epithelial cells to grow over the stroma as in group III, the cells in group I and II have to grow over the cell-fibrin and fibrin matrix, respectively.

Stroma remodeling is a major factor in contributing to the transparency of the cornea. Based on our result, we observed that the MSCs group developed more corneal opacity. This is consistent with other study by Gu et al..$^{5}$ in that they also showed that groups treated with MSCs developed cornea opacity. In contrast, another study by Ye et al. $^{14}$ showed significantly clear corneal in the treated group with MSCs compared to those without MSCs. These differences may be due to different methods of transplantation.

In the present study, we observed that MSCs tend to distribute more at the edge of the defect. The cellular distribution was not even due to the curvature of the stromal defect created. It also explained why the scarring tends to be annular in shape in the treated group. It developed opacity, but from the increase in corneal thickness, a new stromal matrix must have been produced.

The optical coherence tomography (OCT) can visualize the transplanted MSCs within the lamellar dissected cornea and amniotic membrane overlying it. We found that the normal corneal thickness in experimental rabbits range from 408 $\mu \mathrm{m}$ to $342 \mu \mathrm{m}$, which is similar to the findings in other studies.,

We found that the corneal thickness had significantly increased from day 30 to day 60 and at day 60 in group I, the newly regenerated cornea is marked by the presence of a thick stroma layer with an increase in cellular density in the region close to the corneal defect that was created. It showed that MSCs-treated group has participated in stroma regeneration. The difference of corneal thickness in BM-MSCs treated group had significantly increased at day 60 compared to day 30 , indicating that stromal regeneration continues throughout the time frame of this experiment. In groups II and III, corneal thickness also increased at day 30 but had not increased significantly at day 60 . Further, epithelial hyperplasia noted histologically in group II and III. This study also found that stroma took a long time to regenerate as the thickness from postoperatively to day 30 was not significant between all groups.

The present study had the following limitations: transplantation method of MSCs results in excessive accumulation of cells at the edge of the wound and less remaining in the central region. We did not investigate whether the transplanted MSCs differentiated into functional keratocytes or if they exert a paracrine effect in promoting wound healing. Despite the limitations, we showed that transplanted 
BM-MSCs are capable of regenerating corneal stroma. However, this beneficial effect comes at the expense of increased corneal haze.

\section{Conflict of interest}

The authors have no conflict of interest to declare in the conduct of the study.

\section{References}

1. Francisco AM, Silvia P, Alejandro BM, et al. Adipose-derived stem cells are a source for cell therapy of the corneal stroma. Stem Cells 2008;26:570-579.

2. Flavia LB, Shyam SC, Alicia C, et al. Corneal myofibroblast generation from bone marrow-derived cells. Exp Eye Res 2010;91:92-96.

3. Chan T, Payor S, Holden BA. Corneal thickness profiles in rabbits using an ultrasonic pachometer. Invest Ophth Vis Sci 1983;24:1408-1410.

4. Fantes FE, Hanna KD, Waring GO, et al. Wound healing after excimer laser keratomileusis (photorefractive keratectomy) in monkeys. Arch Ophthalmol 1990;108:665-675.

5. Gu S, Xing C, Han J, Tso MOM, Hong J. Differentiation of rabbit bone marrow mesenchymal stem cells into corneal epithelial cells in vivo and ex vivo. Mol Vis 2009;15: 99.

6. Yosuke $\mathrm{H}$, Waka I, Ken F, et al. Identification of keratocyte-like cells differentiated from circulating bone marrow-derived cells in the mouse cornea. Med Mol Morphol 2013;46:233-238.

7. Kamran H, Alexander IK, Irina YP, et al. Monitoring of rabbit cornea response to dehydration stress by optical coherence tomography. Invest Ophth Vis Sci 2004;45:2555-2562.

8. Yanling $M$, Yongsheng $X$, Zhifeng $X$, et al. Reconstruction of chemically burned rat corneal surface by bone marrow-derived human mesenchymal stem cells. Stem Cells 2006;24:315-321.

9. Norzana AG, Ropilah AR, Jemaimah C, et al. Rabbit limbal epithelial cells maintain its stemness in serum-free and feeder layer-free culture system. Tissue Eng Regen Med 2007;4:557-565.

10. Joo YO, Mee KK, Mi SS, et al. The anti-inflammatory and anti-angiogenic role of mesenchymal stem cells in corneal wound healing following chemical injury. Stem Cells 2008;26:1047-1055.

11. Soo HP, Kyoung WK, Yeoun SC, Jae CK. Human mesenchymal stem cells differentiate into keratocyte-like cells in keratocyte-conditioned medium. Exp Eye Res 2012;101:16-26.

12. Rohaina CM, Then KY, Angela NMH, et al. Reconstruction of limbal stem cell deficient corneal surface with induced human bone marrow mesenchymal stem cells on amniotic membrane. Transl Res 2014;163:200-210.

13. Wakitani S, Goto T, Pineda SJ, et al. Mesenchymal cell-based repair of large, full-thickness defects of articular cartilage. J Bone Joint Surg Am 1994;76:579-592.

14. Ye J, Yao K, Kim JC. Mesenchymal stem cell transplantation in a rabbit corneal alkali burn model: engraftment and involvement in wound healing. Eye 2005;20:482-490. 